\title{
Absorption features in the X-shooter GRB afterglow sample
}

\author{
Antonio de Ugarte Postigo ${ }^{1,2}$ for the X-shooter GRB collaboration \\ ${ }^{1}$ Instituto de Astrofísica de Andalucía (IAA-CSIC), Granada, Spain \\ email: deugarte@iaa.es \\ ${ }^{2}$ Dark Cosmology Centre, NBI, U. Copenhagen, Copenhagen Ø, Denmark
}

\begin{abstract}
Since 2009 X-shooter has collected spectroscopy of over 80 gamma-ray burst afterglows with redshifts ranging from 0.05 to 6.3 . Thanks to its efficiency, broad wavelength coverage $(3,000$ to $24,800 \AA)$, and intermediate spectral resolution $(\mathrm{R} \sim 8,000)$ it has become the most efficient tool for gamma-ray burst afterglow spectroscopy. In this Focus Meeting we presented the sample and some preliminary results of the analysis of absorption systems.
\end{abstract}

Keywords. gamma-rays: bursts, astronomical data bases: miscellaneous

\section{The X-shooter GRB afterglow sample}

X-shooter (Vernet et al. 2011) is ESO's 3-arm echelle spectrograph that is working on the Very Large Telescope (Paranal Observatory, Chile). One of its science drivers was the study of gamma-ray burst (GRB) afterglows. Indeed, it has become the world's most prolific spectrograph in the observation of these events, with over 80 observed afterglows.

Our collaboration has used X-shooter to systematically observe all GRBs that had a good observability, defined as follows: The line of sight is not strongly affected by Galactic extinction $\left(\mathrm{A}_{V}<0.5 \mathrm{mag}\right)$, they have a precise X-ray localisation within 10 minutes, and are visible for at least 60 minutes during the night at Paranal. This helps to reduce the bias existent in the overall sample, and allows better statistical studies.

We are working on the analysis of absorption features in a similar way as we did in 2012 for a sample of low resolution spectra (de Ugarte Postigo et al. 2012). With X-shooter, we have a much better coverage of the spectral features thanks to the broad wavelength range (the 22 lines presented in that paper are all covered by an $\mathrm{X}$-shooter spectrum for redshifts between 1.6 and 6.2). Furthermore, with a spectral resolution of $\sim 30 \mathrm{~km} \mathrm{~s}^{-1}$, we can deblend many more lines and even study velocity components.

For this work we have developed an analysis tool to automatically detect, measure, and plot the absorption features. Using this measurements we can perform statistical studies of the different features, comparing them with the properties of the prompt GRB emission, the properties of the afterglow, the associated supernova, or even the host galaxy. The reduced data and the spectral line measurements will be made public through our GRB spectra database: grbspec.iaa.es (de Ugarte Postigo et al. 2014).

\section{References}

de Ugarte Postigo, A et al. 2012, A\& A, 548, 11

de Ugarte Postigo, A et al. 2014, SPIE, 9152, 0

Vernet, J, et al. 2011, A\&BA, 536, 105 